\title{
Covalency-Driven Dimerization of Plutonium(IV) in a Hydroxamate Complex
}

\author{
Mark A. Silver, ${ }^{1}$ Samantha K. Cary, ${ }^{1}$ Jared T. Stritzinger, ${ }^{1}$ T. Gannon Parker, ${ }^{1}$ Laurent Maron, ${ }^{2}$ \\ Thomas E. Albrecht-Schmitt*1 \\ ${ }^{1}$ Department of Chemistry and Biochemistry, Florida State University, 95 Chieftan Way, \\ Tallahassee, Florida 32306, United States \\ ${ }^{2}$ Laboratoire de Physique et Chimie des Nano-Objets, Institut National des Sciences Appliquées, \\ 135 Avenue de Rangueil, 31077 Toulouse Cedex 4, France
}

\section{Electronic Supporting Information}

Crystallographic Data

S 1

Materials and Synthesis

S2-S3

Computational Details, Data, and References

S4-S9 
Table S1: Selected crystallographic information for $\mathrm{Pu}_{2}(\mathrm{FHA})_{8}$.

\begin{tabular}{|c|c|}
\hline Compound & $\mathrm{Pu}_{2}$ (FHA) 8 \\
\hline Formula Mass & 964.29 \\
Color and habit & Red, tablet \\
Space Group & $C 2 / c$ \\
$a(\AA)$ & $12.7319(6)$ \\
$b(\AA)$ & $9.7137(5)$ \\
$c(\AA)$ & $16.9532(8)$ \\
$\alpha\left(^{\circ}\right)$ & 90 \\
$\beta\left(^{\circ}\right)$ & $94.6924(10)$ \\
$\gamma\left({ }^{\circ}\right)$ & 90 \\
$V\left(\AA^{3}\right)$ & $2089.64(18)$ \\
$Z$ & 4 \\
$T(\mathrm{~K})$ & $100(2)$ \\
$\lambda(\AA)$ & 0.71073 \\
Maximum $2 \theta\left(^{\circ}\right)$ & 27.520 \\
$\rho_{\text {calc }}\left(\mathrm{g} / \mathrm{cm}^{3}\right)$ & 3.065 \\
$\mu(\mathrm{Mo} K \alpha)\left(\mathrm{cm}^{-1}\right)$ & 63.51 \\
$R(F)$ for $F_{\mathrm{o}}{ }^{2}>2 \sigma\left(F_{\mathrm{o}}^{2}\right)^{a}$ & 0.0165 \\
$R_{\mathrm{w}}\left(F_{\mathrm{o}}^{2}\right)^{b}$ & 0.0364 \\
\hline
\end{tabular}

${ }^{a} R(F)=\sum|| F_{\mathrm{o}}|-| F_{\mathrm{c}}|| / \sum\left|F_{\mathrm{o}}\right|$

${ }^{b} R_{\mathrm{w}}\left(F_{\mathrm{o}}^{2}\right)=\left[\sum\left[w\left(F_{\mathrm{o}}^{2}-F_{\mathrm{c}}^{2}\right)^{2}\right] / \sum w F_{\mathrm{o}}^{4}\right]^{1 / 2}$ 
Materials and Synthesis. Caution! Inhalation or ingestion of ${ }^{242} \mathrm{Pu}\left(t_{1 / 2}=3.75 \times 10^{5}\right.$ y) represents a potential health risk owing to its $\alpha$ and $\gamma$ emission and the emission of its daughters. ${ }^{242} \mathrm{Pu}$ decays to ${ }^{238} \mathrm{U}\left(\mathrm{t}_{1 / 2}=4.49 \times 10^{9} \mathrm{y}\right)$. All studies were conducted in a radiologic facility. This laboratory is equipped with HEPA filtered hoods and negative pressure gloveboxes. A series of counters continually monitor radiation levels in the laboratory, including air sampling. The laboratory is licensed and inspected by the State of Florida - a Nuclear Regulatory Commissioncompliant state. All experiments were carried out with approved safety operating procedures. All free-flowing solids are worked with in gloveboxes, and products are only examined when coated with either water or Krytox oil and water.

Formohydroxamic acid (FHA) was synthesized using a procedure from Fishbein, W. N., Daly, J., and Streeter, C. L. ${ }^{1}$ Materials needed for the synthesis included hydroxylamine hydrochloride (Sigma-Aldrich, Lot \# 07513DE), ethyl formate (Sigma-Aldrich, Lot \# MKBR0981V), 200 proof pure ethanol (Koptec, Lot \# 115412), ethyl acetate (Macron, Batch \# 0000083641) acetone (BDH, Lot \# 122012A), sodium hydroxide (Fisher Scientific, Lot \# 070241), hydrochloric acid (EMD Chemicals, Lot \# 52139). After securing a $47 \%$ yield of the colorless, crystalline product, ${ }^{1} \mathrm{H}-\mathrm{NMR}$ and ${ }^{13} \mathrm{C}$-NMR analysis were performed using a Bruker Avance III $400 \mathrm{MHz}$ NMR. Deuterium oxide (Cambridge Isotope Laboratories, Lot \# 13G-228) was used to dissolve product in order to obtain the necessary spectra. NMR spectra matched those of PavietHartmann et. al. ${ }^{2}$

$\mathrm{Pu}_{2}$ (FHA)8 was prepared by charging a $20-\mathrm{mL}$ scintillation vial with $25 \mathrm{mg}$ of ${ }^{242} \mathrm{PuI} 3(10 \mathrm{mg}$ ${ }^{242} \mathrm{Pu}$ content $)$, and to it was added $250 \mu \mathrm{L}$ of $1.65 \mathrm{M} \mathrm{FHA}\left(25.2 \mathrm{mg}, 4.13 \times 10^{-4} \mathrm{M}\right)$. This resulted in a dark purple solution. The vial was covered with parafilm and the parafilm was punctured several times to allow the solution to slowly evaporate over a period of $4-6$ weeks. The solution 
slowly transitioned to a dark red color by week 4 , and red crystals precipitated following this phenomenon; the crystals were harvested and rinsed with ethanol. The crystals were weighed after drying to establish yield $(\sim 70 \%)$.

\section{Computational Details.}

All the structures reported in this study were fully optimized with the Becke's 3-parameter hybrid functional ${ }^{3}$ combined with the non-local correlation functional provided by Perdew/Wang ${ }^{4}$ (denoted as B3PW91). The plutonium atom was represented by relativistic energy-consistent small-core pseudopotential obtained from the Stuttgart-Köln ECP library has been used in combination with its adapted segmented basis set. ${ }^{5}$ For the remaining atoms the $6-31 \mathrm{G}(d, p)$ basis set was used. ${ }^{6,7}$ In all computations no constraints were imposed on the geometry. All stationary points have been identified as minima (number of imaginary frequencies $\mathrm{N}_{\mathrm{imag}}=0$ ) or transition states $\left(\mathrm{N}_{\mathrm{imag}}=1\right)$. The vibrational modes and the corresponding frequencies are based on a harmonic force field. Enthalpy energies were obtained at $\mathrm{T}=298.15 \mathrm{~K}$ within the harmonic approximation. GAUSSIAN09 program suite was used in all calculations. ${ }^{8}$ Finally, for the 3D representation of the structures the Chemcraft ${ }^{9}$ program was used as well as for the visualization of the molecular orbitals.

\section{Optimized structure of the $\mathrm{Pu}_{2}$ (FHA) 8}

50

SCF Done: -3063.5884194
C $\quad 35.265548000 \quad-29.439614000 \quad-26.195679000$
C $\quad 33.724151000 \quad-27.940991000 \quad-33.236167000$
C $\quad 36.201367000 \quad-25.824504000 \quad-31.172596000$
$\begin{array}{llll}\text { C } & 32.788332000 & -31.556101000 & -28.259251000\end{array}$
C $\quad 37.038336000 \quad-30.736337000 \quad-30.477520000$ 


\begin{tabular}{|c|c|c|c|}
\hline $\mathrm{C}$ & 31.951362000 & -26.644268000 & -28.954327000 \\
\hline $\mathrm{C}$ & 39.457796000 & -27.230517000 & -28.650599000 \\
\hline $\mathrm{C}$ & 29.531903000 & -30.150088000 & -30.781247000 \\
\hline $\mathrm{H}$ & 35.110219000 & -31.397555000 & -31.022331000 \\
\hline $\mathrm{H}$ & 33.879479000 & -25.983051000 & -28.409515000 \\
\hline $\mathrm{H}$ & 35.881694000 & -24.094829000 & -30.127628000 \\
\hline $\mathrm{H}$ & 33.108004000 & -33.285776000 & -29.304218000 \\
\hline $\mathrm{H}$ & 34.776126000 & -27.833988000 & -25.024776000 \\
\hline $\mathrm{H}$ & 34.213573000 & -29.546617000 & -34.407070000 \\
\hline $\mathrm{H}$ & 40.041422000 & -28.240685000 & -26.965004000 \\
\hline $\mathrm{H}$ & 28.948277000 & -29.139920000 & -32.466842000 \\
\hline $\mathrm{H}$ & 35.002807000 & -30.138286000 & -25.393396000 \\
\hline $\mathrm{H}$ & 33.986892000 & -27.242320000 & -34.038450000 \\
\hline $\mathrm{H}$ & 31.452360000 & -25.755999000 & -28.552004000 \\
\hline $\mathrm{H}$ & 37.537339000 & -31.624606000 & -30.879842000 \\
\hline $\mathrm{H}$ & 36.280998000 & -25.259309000 & -32.109275000 \\
\hline $\mathrm{H}$ & 32.708701000 & -32.121296000 & -27.322571000 \\
\hline $\mathrm{H}$ & 40.477321000 & -26.885730000 & -28.853451000 \\
\hline $\mathrm{H}$ & 28.512378000 & -30.494875000 & -30.578395000 \\
\hline $\mathrm{N}$ & 35.739410000 & -30.685942000 & -30.643269000 \\
\hline $\mathrm{N}$ & 33.250289000 & -26.694664000 & -28.78857800 \\
\hline
\end{tabular}




\begin{tabular}{|c|c|c|c|}
\hline $\mathrm{N}$ & 35.947185000 & -25.103911000 & -30.099353000 \\
\hline $\mathrm{N}$ & 33.042514000 & -32.276694000 & -29.332493000 \\
\hline $\mathrm{N}$ & 35.110412000 & -28.163615000 & -25.920921000 \\
\hline $\mathrm{N}$ & 33.879287000 & -29.216991000 & -33.510925000 \\
\hline $\mathrm{N}$ & 39.285302000 & -27.973396000 & -27.582559000 \\
\hline $\mathrm{N}$ & 29.704397000 & -29.407209000 & -31.849287000 \\
\hline $\mathrm{O}$ & 35.089100000 & -29.609472000 & -30.156137000 \\
\hline $\mathrm{O}$ & 33.900599000 & -27.771133000 & -29.275709000 \\
\hline $\mathrm{O}$ & 35.395712000 & -27.222549000 & -26.835479000 \\
\hline $\mathrm{O}$ & 33.593987000 & -30.158056000 & -32.596367000 \\
\hline $\mathrm{O}$ & 36.339553000 & -27.069204000 & -31.125417000 \\
\hline $\mathrm{O}$ & 32.650146000 & -30.311401000 & -28.306429000 \\
\hline $\mathrm{O}$ & 35.826120000 & -25.678409000 & -28.882881000 \\
\hline $\mathrm{O}$ & 33.163579000 & -31.702196000 & -30.548965000 \\
\hline $\mathrm{O}$ & 38.482018000 & -26.937140000 & -29.393015000 \\
\hline $\mathrm{O}$ & 30.507681000 & -30.443465000 & -30.038831000 \\
\hline $\mathrm{O}$ & 35.687931000 & -29.825338000 & -27.315879000 \\
\hline $\mathrm{O}$ & 33.301768000 & -27.555268000 & -32.115968000 \\
\hline $\mathrm{O}$ & 38.061870000 & -28.417451000 & -27.267465000 \\
\hline $\mathrm{O}$ & 30.927829000 & -28.963154000 & -32.164381000 \\
\hline $\mathrm{O}$ & 37.671174000 & -29.815657000 & -29.894819000 \\
\hline
\end{tabular}



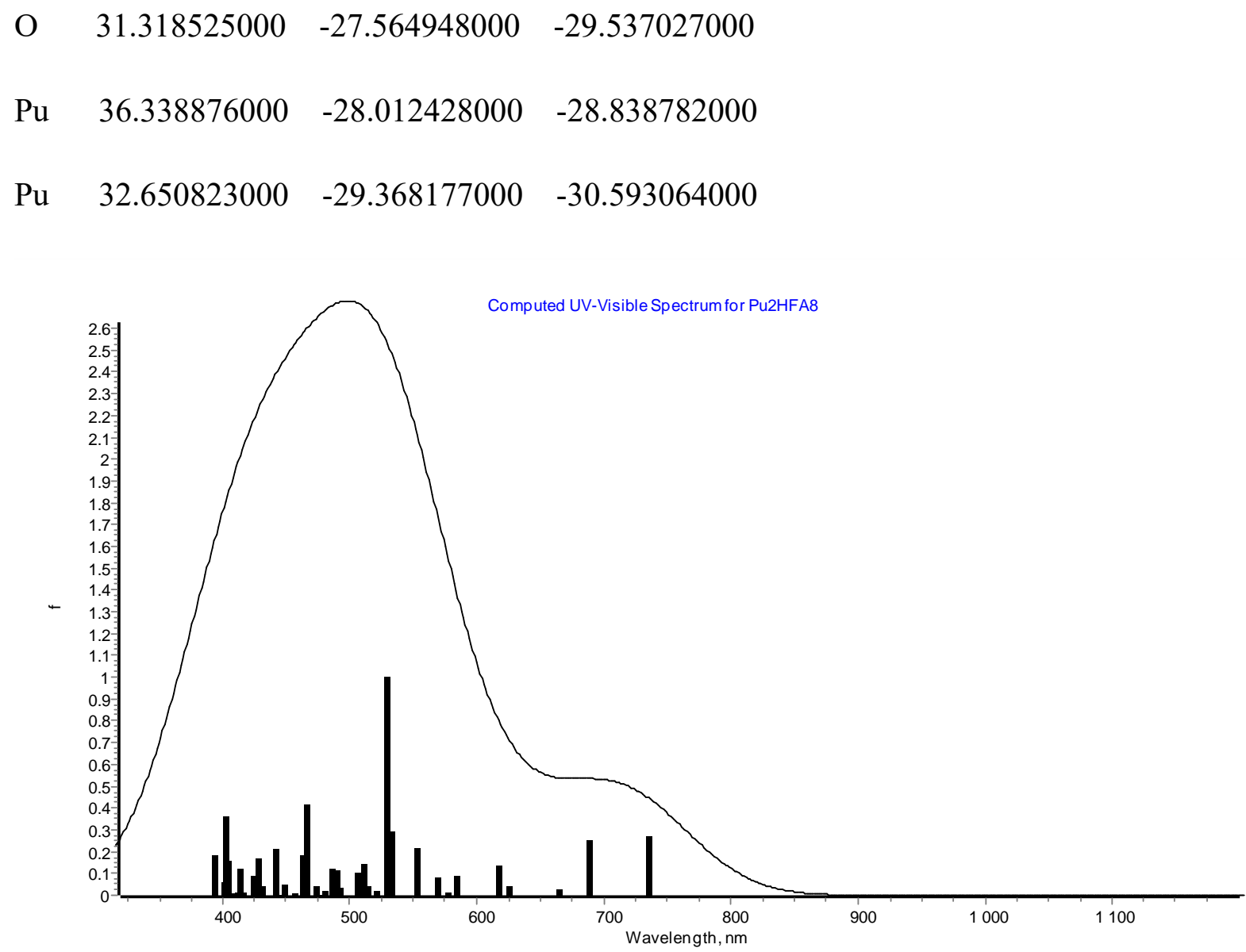

Figure S1: UV-vis-NIR absorption spectrum of $\mathrm{Pu}_{2}(\mathrm{FHA})_{8}$ calculated via TD-DFT quantum mechanical methods

Different spin states were computed from high spin (nonet) to low spin (singlet). The nonet is found to be the lowest state in energy (the singlet being $156 \mathrm{kcal} / \mathrm{mol}$ higher). Time-Dependent density functional theory calculations were carried out to determine the UV-Visible spectrum using the methodology implemented in Gaussian and the lowest 50 states were considered (M. E. Casida, C. Jamorski, K. C. Casida, and D. R. Salahub, J. Chem. Phys., 108 (1998) 4439-49.)

Energy of the different spin states:

$\mathrm{S}=9-3063.5884194$

$\mathrm{S}=7-3063.5387784$

$\mathrm{S}=5-3063.4954997$

$\mathrm{S}=3-3063.4683405$

$\mathrm{S}=1-3063.3397573$ 


\section{References}

1. Fishbein, W. N.; Daly, J.; Streeter, C. L. Anal. Biochem. 1969, 28, 13-24.

2. Paviet-Hartmann, P.; Wright, A.; Mausolf, E.; Campbell, K.; Poineau, F. Proceedings of the $18^{\text {th }}$ International Conference on Nuclear Engineering, Xi'an, China, May 17-21, 2010.

3. Becke, A. D. J. Chem. Phys., 1993, 98, 5648-5652.

4. Perdew, J. P.; Wang, Y. Phys. Rev. B, 1992, 45, 13244-13249.

5. a) Küchle, W.; Dolg, M.; Stoll, H.; Preuß, H. J. Chem. Phys. 1994, 100, 7535-7543; b) Cao, X. Y,; Dolg, M.; Stoll, H. J. Chem. Phys. 2003, 118, 487-497; c) Cao, X.; Dolg, M. J. Molec. Struct. (Theochem) 2004, 673, 203-209.

6. a) Hehre, W. J.; Ditchfield, R.; Pople, J. A. J. Chem. Phys. 1972, 56, 2257-2261; b)

Hariharan, P. C.; Pople, J. A. Theor. Chim. Acta 1973, 28, 213-222.

7. Binkley, J. S.; Pople, J. A.; Hehre, W. J. J. Am. Chem. Soc. 1980, 102, 939-947.

8. Gaussian 09, Revision A.02, Frisch, M. J.; Trucks, G. W.; Schlegel, H. B.; Scuseria, G. E.;

Robb, M. A.; Cheeseman, J. R.; Scalmani, G.; Barone, V.; Mennucci, B.; Petersson, G. A.; Nakatsuji, H.; Caricato, M.; Li, X.; Hratchian, H. P.; Izmaylov, A. F.; Bloino, J.; Zheng, G.;

Sonnenberg, J. L; Hada, M.; Ehara, M.; Toyota, K.; Fukuda, R.; Hasegawa, J.; Ishida, M.; Nakajima, T.; Honda, Y.; Kitao, O.; Nakai, H.; Vreven, T.; Montgomery, Jr., J. A.; Peralta, J. E.; Ogliaro, F.; Bearpark, M.; Heyd, J. J.; Brothers, E.; Kudin, K. N.; Staroverov, V. N.; Kobayashi, R.; Normand, J.; Raghavachari, K.; Rendell, A.; Burant, J. C.; Iyengar, S. S.; Tomasi, J.; Cossi, M.; Rega, N.; Millam, J. M.; Klene, M.; Knox, J. E.; Cross, J. B.; Bakken, V.; Adamo, C.; Jaramillo, J.; Gomperts, R.; Stratmann, R. E.; Yazyev, O.; Austin, A. J.; Cammi, R.; Pomelli, C.; Ochterski, J. W.; Martin, R. L.; Morokuma, K.; Zakrzewski, V. G.; Voth, G. A.; Salvador, P.; 
Dannenberg, J. J.; Dapprich, S.; Daniels, A. D.; Farkas, O.; Foresman, J. B.; Ortiz, J. V.; Cioslowski, J.; Fox, D. J. Gaussian, Inc., Wallingford CT, 2009.

9. Zhurko, G. A. ChemCraft Home Page: a set of graphical tools for facilitating working with quantum chemistry computations (http://www.chemcraftprog.com). 